\title{
Violencia de la mujer hacia el hombre, ¿mito o realidad?
}

\author{
Violence of women againt men, myth or reality?
}

Adrián Aguilera Jiménez, María Barba Priego, Marta Fuentes Gutiérrez, Elena López Molina, Nerea María Villacreces Flores. Grado de Educación Social. Universidad de Granada.

José Miguel García Ramírez. Departamento de Psicología Social. Universidad de Granada.

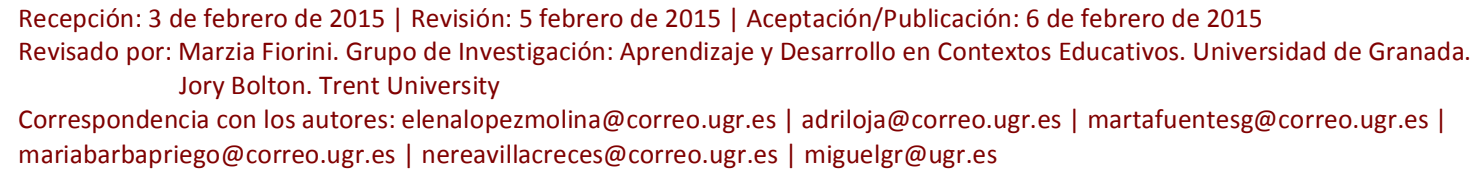

\section{Resumen}

El sexismo ambivalente afectan de manera inmediata y permanente interfiriendo en la necesidad de cierre cognitivo, generando confusión, ambigüedad e incertidumbre en las personas victimas de la violencia de género. Este estudio explora la visión de que las mujeres puedan ejercer la violencia hacia los hombres. Método: Estudio exploratorio realizado en la Facultad de Ciencias de la Educación de la Universidad de Granada. Cuarenta y nueve mujeres, cumplimentaron el Inventario de Sexismo Ambivalente y la escala de Necesidad de Cierre Cognitivo. Resultados: El análisis estadístico muestra que no existen correlaciones significativas entre los resultados obtenidos. Conclusiones: La violencia de las mujeres hacia los hombres no es reconocida.

Palabras clave: Sexismo | Cierre cognitivo

\begin{abstract}
Sexism affects victims in an immediate and permanent way, interfering with the need for cognitive closure and generating confusion, ambiguity and uncertainty in people victimized by gender-based violence. This study explores the idea that women can exercise violence against men. Method: Exploratory study carried out at the Faculty of Education of the University of Granada. Forty-nine women completed the Ambivalent Sexism Inventory and the Need for Cognitive Closure scale. Results: The statistical analysis shows that there is no significant correlation between the results obtained. Conclusions: Violence by women against men is not recognized.
\end{abstract}

Keywords: Sexism | Cognitive Closure

\section{Introducción}

La violencia de las mujeres hacia los hombres en la pareja es un tema poco investigado, a penas hay estudios realizados sobre este fenómeno. Aunque los factores socioculturales son los que influyen en la aparición de la violencia, es la legislación la que protege los derechos por igual de todas las personas; sin embargo existe diferencia penal en los delitos y las penas son imputadas según el género, aunque la ley debiera proteger a las persona con independencia del género, para evitar una discriminación positiva perpetúa. La conducta violenta de mayor impacto es el homicidio, generalmente, perpetrado por hombres; aunque, también hay casos de violencia perpetrados por mujeres directa o indirectamente. En España no existen estudios representativos para generalizar este último dato, ya que la mayoría de los casos las victimas son mujeres (Toldos, 2013). 
La violencia conyugal es una conducta abusiva que se da dentro de una relación que provoca tanto daño físico como psicológico. Generalmente, son formas de demostrar dominación y control sobre la otra persona, que se repite reiteradamente agravándose la intensidad y frecuencia. La mayoría de los investigadores se han centrado en estudiar las características psicológicas y el perfil tanto de las personas agresoras como de las víctimas. Asegurando que una persona maltratadora puede pertenecer a cualquier clase social, con una baja autoestima y un deseo irracional de dominar a la otra persona (Gálvez, 2011). Además desarrollan esa tendencia mediante aprendizaje, ya que la violencia es aprendida, sobre todo, al observar la conducta violenta en modelos referenciales. Por lo tanto, la conducta violenta en el hogar es el resultado de un estado emocional intenso que interactúa con unas actitudes de hostilidad, un repertorio pobre de conductas y unos factores precipitantes, así como la percepción de vulnerabilidad de la víctima (Echeburúa y De Corral, 2009).

La violencia doméstica proviene de estructuras sociales, económicas, políticas y culturales desigualitarias e injustas. No se trata de un fenómeno nuevo, pero su reconocimiento como problema social si es relativamente reciente. Para que la violencia ejercida contra las mujeres en el hogar haya trascendido desde el ámbito privado al ámbito público, ha sido necesario que las mujeres paguen un alto precio, pues han sido numerosas las victimas que han muerto a causa de este tipo de violencia (Alonso, Manso, y Sánchez, 2013). Tras más de dos décadas de activismo independiente buscando la atención sobre este problema, los colectivos feministas, consiguieron que los organismos internacionales adquiriesen conciencia de la relevancia de este grave problema (Jiménez, Luengo, y Taberner, 2011). A partir del año 2004, en España, se aprueba una ley específica que tipifica como delito cualquier maltrato familiar, antes estos actos eran considerados como faltas, mientras que a partir de ese año pasa a ser delito por lesión, en el que incluye maltrato físico y psicológico (Maqueda Abreu, 2006). Pero la violencia en el seno de la familia sigue estando oculta, ya que los actos violentos se realizan en la intimidad, dificultándose su detección (Sarasua, y Zubizarreta, 2000).

La tendencia a valorar a las personas según su género presupone sentimientos, emociones, conductas o actividades que se pueden desempeñar, dando lugar a los estereotipos. Actualmente se han reducido las manifestaciones sexistas, pero ha aparecido el sexismo ambivalente, el cual se constituye de dos componentes: hostil y benévolo. Ambos tipos de sexismo se centran en el poder que quieren ejercer o imponer los hombres sobre las mujeres (Expósito, Moya y Glick, 1998). En una investigación reciente se comprobó que los efectos del sexismo ambivalente afectan de manera inmediata y permanente interfiriendo en la necesidad de cierre cognitivo; generalmente las personas buscan una respuesta definitiva ante un problema determinado; el cierre cognitivo evita la confusión, la ambigüedad y la incertidumbre, por lo tanto, puede tener un papel importante en las relaciones intrapersonales, interpersonales, intragrupales e intergrupales (Arjona, y García, 2014). En los casos de violencia en la pareja donde el hombre es la víctima, los medios de comunicación suelen tratar el tema como si fuese un crimen, pero sin hacer mención a los términos de violencia doméstica o abuso por parte de la pareja o violencia de género. Se tiende a cerrar los casos de manera inmediata y permanente como si no hubiesen sucedido. Existen algunos casos de hombres muertos y maltratados por sus parejas o exparejas; sin embargo no han recibido atención por los medios de comunicación. Incluso los hombres, víctimas de la violencia por parte de sus parejas, no son conscientes de que tienen un problema, ya que socioculturalmente la mujer sólo ejerce la violencia para defenderse del hombre. La sociedad actual no da cabida a la existencia del hombre maltratado ya que no existe la visión del hombre maltratado en la violencia doméstica. Es extraño pensar que puedan haber hombre que sean víctimas de malos tratos por parte de sus parejas (Toldos, 2013). 
Con este estudio de carácter exploratorio se pretende abrir una línea de investigación en el ámbito de la violencia de género que sea inclusiva de ambos géneros. Por lo tanto el objetivo es: comprobar si existe consciencia de que la violencia de género puede darse de la mujer hacia el hombre.

\section{Método}

\section{Participantes}

Cuarenta y nueve mujeres, de entre 18 y 50 años $(M=27, S D=7.8)$. La muestra fue escogida aleatoria en la Facultad de Ciencias de la Educación de la Universidad de Granada.

\section{Instrumentos}

Se utilizaron el Inventario de Sexismo Ambivalente, ASI, (Expósito, Moya, y Glick, 1998) y el test de Necesidad del Cierre Cognitivo, NCC, (Horcajo, Díaz, Gandarillas, y Briñol, 2011). La escala de sexismo ambivalente consta con 22 ítems, con 6 respuestas establecidas que van desde "totalmente en desacuerdo" hasta "totalmente de acuerdo". El Test de Necesidad de Cierre Cognitivo consta de 14 ítems con un intervalo de respuestas igual que la escala de sexismo. Los análisis de fiabilidad de ambos instrumentos fueron de un Alfa de Cronbach de 0.791 para la escala de Necesidad de Cierre Cognitivo y un Alfa de Cronbach de 0.835 para el Inventario de Sexismo Ambivalente.

\section{Procedimiento}

Los instrumentos fueron cumplimentados por 49 mujeres el día 7 de abril en la cafetería de la Facultad de Ciencias de la Educación de la Universidad de Granada. Se les pidió que asumieran el rol de hombre antes de completar ambas escalas. Posteriormente los datos obtenidos se analizaron estadísticamente con el programa de estadística SPSS 20.

\section{Resultados}

A los datos obtenidos se les realizó un análisis estadístico de correlaciones de Pearson para comprobar el grado de correlación y significancia entre las diferentes variables, el resultado mostró que correlacionan significativamente las variables de cada instrumento utilizado, pero éstas no correlacionan con las variables del otro instrumento, tal como se muestra en la Tabla 3; por lo tanto no se puede demostrar que exista relación entre el sexismo ambivalente y la necesidad de cierre cognitivo.

\begin{tabular}{|c|c|c|c|c|}
\hline \multicolumn{5}{|c|}{$\begin{array}{l}\text { Tabla } 3 \\
\text { Correlaciones de Pearson del ISA y la NCC }\end{array}$} \\
\hline & Urgencia & Permanencia & Benevolente & Hostil \\
\hline Urgencia & 1 & & & \\
\hline Permanencia &, $398^{\wedge}$ & 1 & & \\
\hline Benevolente &,- 151 & ,077 & 1 & \\
\hline Hostil &,- 013 &, 174 &, $385^{\wedge}$ & 1 \\
\hline
\end{tabular}




\section{Discusión y conclusiones}

El hecho no se correlacionen las variables del ASI con las NCC puede interpretarse como la ausencia de consciencia por parte de las mujeres de ella puedan ejercer la violencia hacia el hombre, por lo tanto no tienen necesidad de cierre cognitivo. Además ella entienden como violencia de género la que ejerce el hombre sobre la mujer. Por lo tanto este estudio exploratorio muestra que la violencia de las mujeres hacia los hombres no es reconocida, posiblemente debido a la falta de conciencia o a la escasez.

Para concluir, la violencia de género de las mujeres hacia los hombres, aunque sea escasa, existe y sería necesario investigarla porque la sociedad se debe construir desde la igualdad.

\section{Referencias}

Alonso, M.B., Manso, J.M., y Sánchez, M.E. (2013). Revisión teórica del maltrato psicológico en la violencia conyugal. Psicología y Salud, 20(1), 65-75. http://revistas.uv.mx/index.php/psicysalud/article/view/618

Arjona Martin, N., y Garcia-Ramirez, JM. (2014). Los efectos del sexismo no se olvidan ni inmediatamente ni permanentemente. ReiDoCrea, 3, 267-272. http://hdl.handle.net/10481/32861

Echeburúa, E., y De Corral, P. (2009). Manual de violencia familiar. Madrid: Siglo XXI. ISBN 13: 9788432309717.

Expósito, F., Moya, M., y Glick, P. (1998). Sexismo ambivalente: medición y correlatos. Revista de Psicología Social, 13(2), 159-169. http://dx.doi.org/10.1174/021347498760350641

Gálvez Montes, C. (2011). Violencia de género: Terrorismo en casa. Jaen: Formación Alcalá. ISBN 13: 9788499763385.

Jiménez, M., Luengo, J. y Taberner, J. (2009). Exclusión social y exclusión educativa como fracasos. Conceptos y líneas para su comprensión e investigación. Profesorado. Revista de Curriculum y Formación del Profesorado, 13(3), 11-49. http://helvia.uco.es/xmlui/handle/10396/8647

Horcajo, J., Díaz, D., Gandarillas, B., y Briñol, P. (2011). Adaptación al castellano del Test de Necesidad de Cierre Cognitivo. Psicothema, 23(4), 864-870. http://www.unioviedo.es/reunido/index.php/PST/article/view/9171

Maqueda Abreu, M.L. (2006). La violencia de género: Entre el concepto jurídico y la realidad social." Revista Electrónica de Ciencia Penal y Criminología 8(2), 1-13. http://criminet.ugr.es/recpc/08/recpc08-02.pdf

Sarasua, B. y Zubizarreta, I. (2000). Violencia en la pareja. Málaga: Ediciones Aljibe. ISBN 13: 9788495212870.

Toldos, M. (2013). Hombres víctimas y mujeres agresoras. La cara oculta de la violencia entre sexos. Córdoba: Editorial Cántico. ISBN 13: 9788494036897. 